\section{CASE REPORT}

\author{
J.I. Berman \\ S.E.G. Hamrick \\ P.S. McQuillen \\ C. Studholme \\ D. $\mathrm{Xu}$
}

R.G. Henry

L.K. Hornberger

O.A. Glenn

\title{
Diffusion-Weighted Imaging in Fetuses with Severe Congenital Heart Defects
}

SUMMARY: Fetal diffusion MR imaging was performed in 3 fetuses with CHD. ADC values in the periatrial WM, thalamus, and basal ganglia were compared with those in a control population of fetuses. Diffusivity in the periatrial WM and thalamus was higher for the fetuses with CHD compared with controls. These observations support the finding of abnormal in utero brain development in fetuses with $\mathrm{CHD}$.

ABBREVIATIONS: $A D C=$ apparent diffusion coefficient; $C H D=$ congenital heart disease; $W M=$ white matter

C ongenital heart anomalies have been linked to long-term motor, sensory, and cognitive difficulties. ${ }^{1,2}$ The origin and cause of these functional deficits are not entirely understood. Abnormal WM structure associated with CHD has been detected with MR imaging at an early stage of neurodevelopment in neonates before corrective surgery. ${ }^{3}$ This study sought to determine the feasibility of detecting structural abnormalities in utero with diffusion MR imaging. The diffusion of water is sensitive to subtle changes in WM architecture that may not be visible on traditional MR imaging. This study measured diffusivity in the basal ganglia, thalamus, and periatrial WM of 3 fetuses with CHD. Diffusion measurements were compared with those in a population of control fetuses. ${ }^{4}$

\section{Case Reports}

Three women were referred for MR imaging of the fetal brain after prenatal sonography showed congenital cardiac anomalies. All fetal brain MR imaging was performed as part of a study on CHD, which was approved by our institutional review board. Case 1 was a fetus at 32.14 weeks' gestational age at MR imaging with sonographic evidence of transposition of the great arteries, mild hydronephrosis, and a small cystic neck mass. T1- and T2-weighted imaging findings of the fetal brain were normal. Case 2 was a fetus at 34.57 weeks' gestational age with a hypoplastic left heart and mild pelviectasis on prenatal sonography. No abnormalities were evident on T1- or T2-weighted MR images. Case 3 was a fetus at 35.29 weeks' gestational age with a hypoplastic left heart identified on prenatal sonography. Fetal MR imaging demonstrated a hypoplastic corpus callosum. In Cases 1 and

Received October 16, 2009; accepted after revision October 20.

From the Departments of Radiology and Biomedical Imaging (J.I.B., C.S., D.X., R.G.H., O.A.G.) and Pediatrics (P.S.M.), University of California, San Francisco, San Francisco, California; Department of Pediatrics (S.E.G.H.), Emory University, Atlanta, Georgia; and Department of Pediatrics (L.K.H.), University of Alberta, Edmonton, Alberta, Canada.

This work was supported by the Dana Foundation Brain and Immuno Imaging Track A, National Institutes of Health grant K23 NS52506, and NIH/NCRR UCSF-CTSI grant UL1 RR024131. Its contents are solely the responsibility of the authors and do not necessarily represent the official views of the NIH.

Please address correspondence to Orit A. Glenn, MD, UCSF, Department of Radiology, 505 Parnassus Ave, Box 0628, San Francisco, CA 94143; email: orit.glenn@radiology.ucsf.edu

Indicates open access to non-subscribers at www.ajnr.org

DOI 10.3174/ajnr.A1975
2, a neonatal MR imaging was also performed, which demonstrated a normal appearance of the brain.

Diffusion-weighted imaging was performed on a $1.5 \mathrm{~T}$ scanner with a b-value of $600 \mathrm{~s} / \mathrm{mm}^{2}$ applied in 3 orthogonal directions in addition to a single $b=0 \mathrm{~s} / \mathrm{mm}^{2}$ volume. Imaging parameters were $\mathrm{TR}=4500 \mathrm{~ms}, \mathrm{TE}=$ minimum, FOV $=32 \times 32 \mathrm{~cm}$, section thickness $=5 \mathrm{~mm}$, and matrix $=128 \times 128$. To minimize motion artifacts, we acquired diffusion images during a maternal breath-hold. All acquired echo-planar images and the calculated ADC maps were visually examined, and those degraded by motion were excluded. Regions of interest were placed in the periatrial WM, thalamus, and basal ganglia, and ADC values were measured.

Fetuses with CHD were compared with a previously reported control fetal population comprising 28 fetuses with basal ganglia measurements, 33 fetuses with periatrial WM measurements, and $28 \mathrm{fe}$ tuses with thalamic measurements. ${ }^{4}$ The control population's gestational age at the time of the MR imaging ranged from 21 to 34 weeks. Statistical comparisons between the control population and the 3 fetuses with CHD were made with linear regression, including $\mathrm{ADC}$ and gestational age as variables. In addition, $z$ scores of the individual patient measurements were calculated to determine their deviation from the control data.

$\mathrm{ADC}$ values in the periatrial WM and thalamus were higher for all fetuses with CHD compared with the control population (Fig 1). The average $z$ score was 1.26 for the periatrial WM, 1.38 for the thalamus, and 0.40 for the basal ganglia (Fig 2). Regression analysis indicated a significant difference between patient and control thalamic ADC values $(P<.05)$ and a strong trend not reaching significance between patient and control periatrial WM ADC values $(P=.071)$. Patient and control basal ganglia ADC values were not significantly different $(P=$ $.53)$.

\section{Discussion}

We used fetal diffusion MR imaging to detect abnormally high water diffusion in the thalamus and periatrial WM of fetuses with CHD. High diffusivity indicates a difference in the structural organization of macromolecules during a critical period of neurodevelopment. Certain congenital heart lesions cause changes in cerebral perfusion and/or cerebral hypoxia, potentially leading to abnormal brain development and injury. ${ }^{5-7}$ This study reinforces the hypothesis that brain development is affected in utero by CHD. 

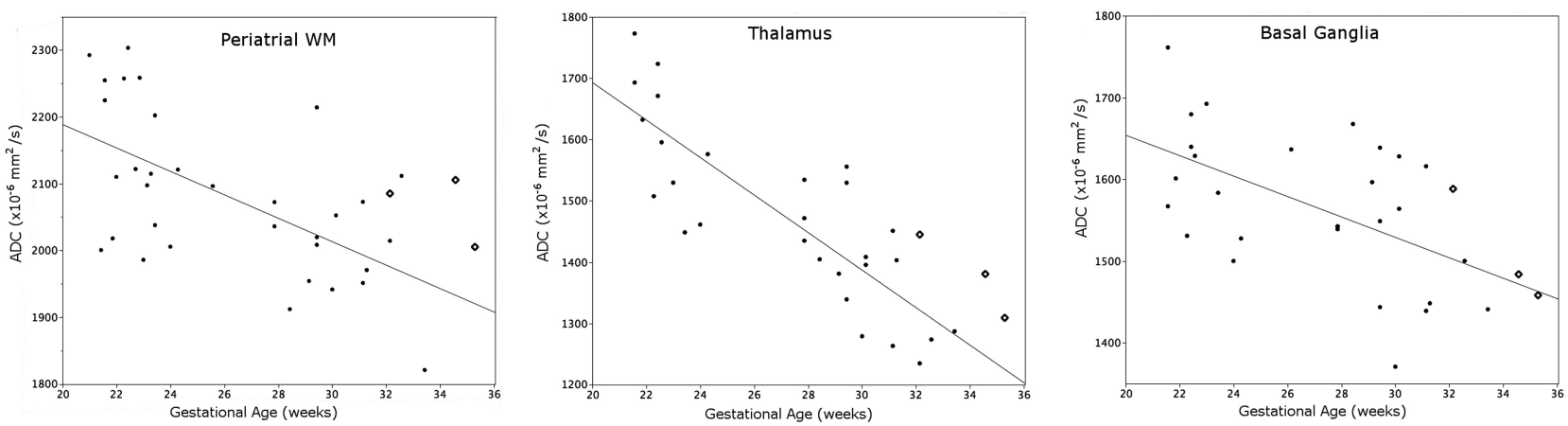

Fig 1. Three fetuses with congenital heart disease (diamonds) are compared with a control cohort (solid dots). The regression line was fit to the control population

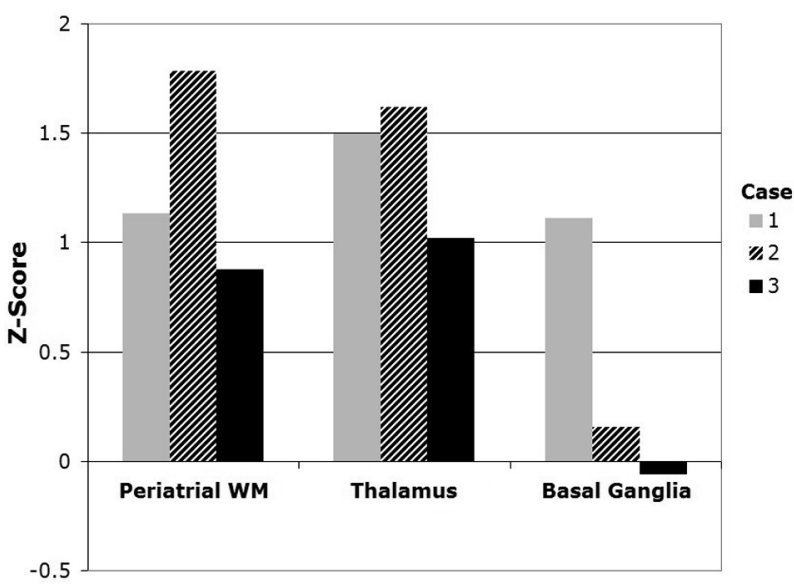

Fig 2. Z scores of individual $A D C$ measurements in fetuses with $C H D$ show deviation from the control population. A negative $z$ score indicates that the ADC measurement was below the expected value from the control population.

In a recent study of neonates with $\mathrm{CHD}$, Miller et $\mathrm{al}^{3} \mathrm{ob}-$ served abnormally high diffusion in the neonatal brain before corrective heart surgery. In particular, the thalamus, perirolandic WM, posterior WM, and optic radiations appear to be most affected in neonates with $\mathrm{CHD} .{ }^{3}$ We also observed abnormal diffusion in the thalamus and posterior WM of the fetuses in our study. We did not observe a significant abnormality in basal ganglia diffusivity in our patients; these results are similar to those of Miller et al. It is not precisely known why particular brain structures may be more susceptible to delayed development or damage in patients with CHD. However, dif- ferent regions of the brain develop at different rates, and perfusion levels can vary throughout the brain. These developmental and anatomic differences may make certain parts of the brain more susceptible to damage and delayed development stemming from decreased perfusion in utero.

These case reports show that fetal diffusion MR imaging is a feasible method of detecting abnormal brain diffusivity associated with CHD. Further studies by using advanced in utero MR imaging techniques such as diffusion tensor imaging, spectroscopy, and possibly functional MR imaging may answer questions concerning the early structural and functional impact of CHD on the developing brain.

\section{References}

1. Bellinger D, Jonas R, Rappaport L, et al. Developmental and neurologic status of children after heart surgery with hypothermic circulatory arrest or lowflow cardiopulmonary bypass. N Engl J Med 1995;332:549-55

2. Partridge S, Vigneron D, Charlton N, et al. Pyramidal tract maturation after brain injury in newborns with heart disease. Ann Neurol 2006;59:640-51

3. Miller S, McQuillen P, Hamrick S, et al. Abnormal brain development in newborns with congenital heart disease. N Engl J Med 2007;357:1928-38

4. Schneider M, Berman J, Baumer F, et al. Normative apparent diffusion coefficient values in the developing fetal brain. AJNR Am J Neuroradiol 2009;30: 1799-803. Epub 2009 Jun 25

5. Donofrio M, Bremer Y, Schieken R, et al. Autoregulation of cerebral blood flow in fetuses with congenital heart disease: the brain sparing effect. Pediatr Cardiol 2003;24:436-43

6. Licht DJ, Wang J, Silvestre DW, et al. Preoperative cerebral blood flow is diminished in neonates with severe congenital heart defects. J Thorac Cardiovasc Surg 2004;128:841-49

7. Shillingford AJ, Ittenbach RF, Marino BS, et al. Aortic morphometry and microcephaly in hypoplastic left heart syndrome. Cardiol Young 2007;17:189-95. Epub 2007 Mar 5 